23

\title{
Сравнительный анализ чувствительности оптических датчиков на поверхностных волнах, возбуждаемых по схеме Кречмана
}

\author{
(C) А.Б. Петрин \\ Объединенный институт высоких температур РАН, \\ 125412 Москва, Россия \\ e-mail: a_petrin@mail.ru \\ Поступила в редакцию 14.05.2019 г. \\ В окончательной редакции 30.07.2019 г. \\ Принята к публикации 12.08.2019 г.
}

На основе теории отражения ограниченного светового пучка от плоскослоистой структуры рассмотрены возбуждаемые по схеме Кречмана поверхностная плазмонная волна на свободной поверхности пленки золота, а также для сравнения поверхностная волна в планарном поверхностном волноводе. Сравнивается чувствительность оптических датчиков на основе указанных поверхностных волн. Предложены различные способы увеличения чувствительности датчиков на поверхностном волноводе. Оценена необходимая точность изготовления толщин пленок для получения работоспособных датчиков.

Ключевые слова: поверхностные волны, поверхностные плазмоны, интегральная оптика, оптические сенсоры.

DOI: $10.21883 /$ OS.2019.12.48708.160-19

\section{Введение и постановка задачи}

Многоканальные оптические сенсорные системы являются ключевой частью современных биологических, химических и физических исследовательских установок [1-4]. Каждый канал в таких системах является датчиком изменения показателя преломления в тонком поверхностном слое металлической пленки, по границе которой распространяются поверхностные плазмонные волны $[5,6]$. Для возбуждения поверхностных плазмонных волн часто используется метод Кречмана [7]. В рамках схемы Кречмана [8] (рис. 1) согласование по волновому числу поверхностной плазмонной волны 1 на поверхности металлической пленки 2 и падающего волнового пучка 3 осуществляется с помощью стеклянной призмы 4.

Линейно поляризованный монохроматический волновой пучок $p$-поляризации падает на металлическую пленку со стороны призмы. Вектор электрического поля падающей волны направлен в плоскости падения. Волновое число поверхностной плазмонной волны больше волнового числа плоской волны в свободном пространстве той же частоты $[9,10]$. Чтобы обеспечить равенство волновых чисел падающей 3 , отраженной 5 и поверхностной плазмонной 1 волн вдоль поверхности металла (рис. 1), необходимо, чтобы падающая волна подходила к пленке из оптически более плотной среды (где волновое число больше, чем в свободном пространстве). Показатель преломления призмы $n_{p}$ и оптимальный угол падения $\alpha_{\mathrm{opt}}$ подбираются таким образом, чтобы обеспечивалось равенство волновых чисел падающей и поверхностных волн. При этих параметрах в отсутствие металличе- ской пленки (и возбуждения поверхностных плазмонов) наблюдается полное внутреннее отражение падающей волны.

Эксперименты показывают, а теория отражения плоских электромагнитных волн от слоистых сред это подтверждает, что при угле падения $\alpha_{\text {орt}}$, соответствующем согласованию по волновому числу падающей и поверхностной плазмонной волн, наблюдается резкое падение коэффициента отражения. Так, при длине падающей волны в вакууме $\lambda=633 \mathrm{~nm}$ и толщине пленки золота, приблизительно равной $48.6 \mathrm{~nm}$ (показатель преломления призмы $n_{p}=1.6$, свободного пространства $n_{s}=1$ ), расчеты показывают сильное изменение коэффициента отражения практически от единицы до нуля в окрестности угла падения, соответствующего согласованию свободной и поверхностной плазмонной волн $\alpha_{\mathrm{opt}}=40.98^{\circ}$.

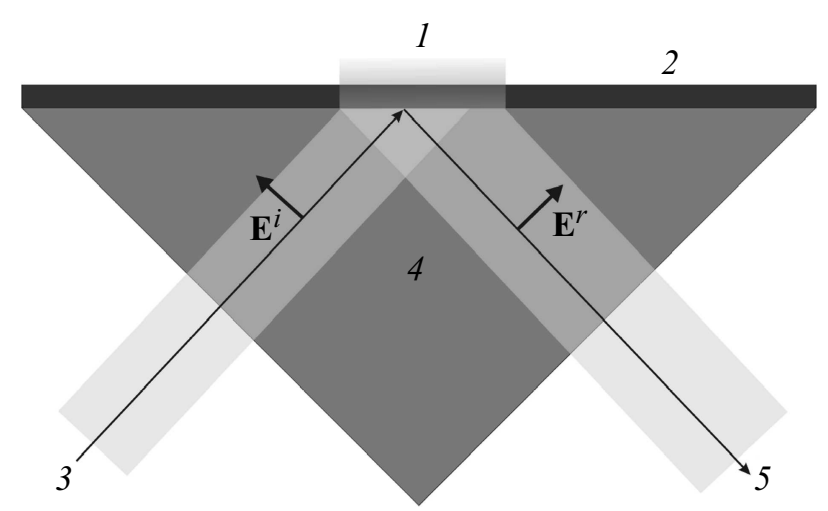

Рис. 1. Схема возбуждения поверхностной плазмонной волны 1 на поверхности металлической пленки 2 по схеме Кречмана. Падающая волна 3 в призме 4 порождает поверхностную плазмонную волну 1 и отраженную волну 5 . 
Таблица 1

\begin{tabular}{c|c|c|c|c|c}
\hline & $\begin{array}{c}\text { Показатель } \\
\text { призмы } \\
\text { призмы }\end{array}$ & $\begin{array}{c}\text { Диэлектрическая проница- } \\
\text { емость золотой пленки }\end{array}$ & $\begin{array}{c}\text { Показатель преломления } \\
\text { свободного пространства }\end{array}$ & $\begin{array}{c}\text { Оптимальная толщина } \\
\text { золотой пленки }\end{array}$ & $\begin{array}{c}\text { Угол наименьшень } \\
\text { отражения, } \\
\mathrm{deg}\end{array}$ \\
\hline $\begin{array}{c}\text { Призма+золотая } \\
\text { пленка+воздуха }\end{array}$ & $n_{p}=1.6$ & $\varepsilon_{m}=-11.6+i 1.2$ & $n_{s}=1$ & $48.6 \mathrm{~nm}$ & 40.980 \\
\hline $\begin{array}{c}\text { Призма+золотая } \\
\text { пленка+вода }\end{array}$ & $n_{p}=1.6$ & $\varepsilon_{m}=-11.6+i 1.2$ & $n_{s}=1.33$ & $48.5 \mathrm{~nm}$ & 64.474
\end{tabular}

При небольшом отклонении угла падения от угла согласования коэффициент отражения возвращается практически от нуля к единичному значению. Указанный угол оптимального возбуждения поверхностной волны зависит от показателей преломления призмы, пленки и свободного пространства за пленкой (рис. 1). В табл. 1 приведены теоретические значения оптимальных значений толщины пленки золота и соответствующие им значения углов наименьшего отражения для свободного пространства, заполненного воздухом и водой. Метод расчета углового распределения коэффициента отражения подробно изложен в работе [11]. Метод нахождения оптимальной толщины пленки заключался в численном варьировании с малым шагом толщины пленки и нахождении такого значения толщины, при котором угловое распределение коэффициента отражения имеет самый глубокий минимум [12]. Эту толщину пленки будем называть оптимальной толщиной, а соответствующий этой толщине угол минимального отражения - оптимальным углом.

Поле поверхностной плазмонной волны сосредоточено в тонком слое вблизи поверхности металла, поэтому характер отражения в схеме Кречмана сильно зависит от изменений показателя преломления в тонком слое вблизи поверхности. Именно на этом основывается широкое применение поверхностных плазмонных волн для создания различного рода датчиков, обладающих высокой чувствительностью [13]. Исследования показали, что чувствительность таких датчиков определяется угловой шириной минимума отражения [14], которая, в свою очередь, определяется поглощением в металле пленки. Чем меньше поглощение в металле, тем меньше угловая ширина минимума и выше чувствительность.

Возбуждение поверхностной плазмонной волны в схеме Кречмана исследовалось для случая, когда апертура падающего светового пучка ограничена. Оказалось, что при этом отражение будет происходить не совсем так, как в случае идеально плоских волн. Так, в работе [11] было показано, что и в случае ограниченной апертуры падающей волны острый минимум отражения (при $\alpha_{\text {opt}}$ ) наблюдается, но отраженные волны имеют угловое распределение в пределах угла дифракции. Дальнейшее исследование отраженных и поверхностных волн вблизи пленки [15] показало, что падающий линейно $p$ поляризованный световой пучок ограниченной апертуры после отражения разделяется на две интерферирующих волны, а поверхностная волна растет в пределах области падения пучка и далее убывает вдоль поверхности [16].

Известно, что поверхностные волны могут распространяться и в диэлектрических [17], и комбинированных металлодиэлектрических [18] пленках планарных волноводов. В связи с этим было предложено $[12,19]$ по схеме Кречмана возбуждать поверхностную волну в многослойной чисто диэлектрической структуре, представляющий из себя планарный волновод. Так, в [12] было предложено использовать пленки с известным заметным затуханием таким образом, чтобы их толщина определяла затухание поверхностной волны, распространяющейся в составном многослойном волноводе. Этим приемом было преодолено ограничение чувствительности датчиков на поверхностных плазмонах металлических пленок, в которых проводимость металла определяла затухание поверхностной плазмонной волны. Теоретически было показано [12], что можно создать многослойную волноводную структуру и датчик на его основе с намного большей чувствительностью по сравнению с датчиками на поверхностных плазмонных волнах на границах металлических пленок.

Однако в [12] не был рассмотрен вопрос о возможной цене увеличения чувствительности предложенных датчиков на поверхностных многослойных волноводах. В частности, не возрастают ли чрезмерно требования к точности изготовления толщин пленок и к размерам датчиков в плоскости поверхностных волноводов? Далее на конкретных примерах будут даны ответы на эти и другие связанные с ними вопросы.

Отметим, что ниже будут приведены только результаты расчетов, детали которых применительно к проблемам настоящей работы подробно изложены в работах $[11,15,16]$. Поэтому ради экономии места и ухода от повторений детали расчетов далее опускаются.

\section{Оценка чувствительности датчика на поверхностной плазмонной волне по границе пленки золота}

Для оценки чувствительности рассмотрим случай, когда датчик состоит из призмы, золотой пленки и 


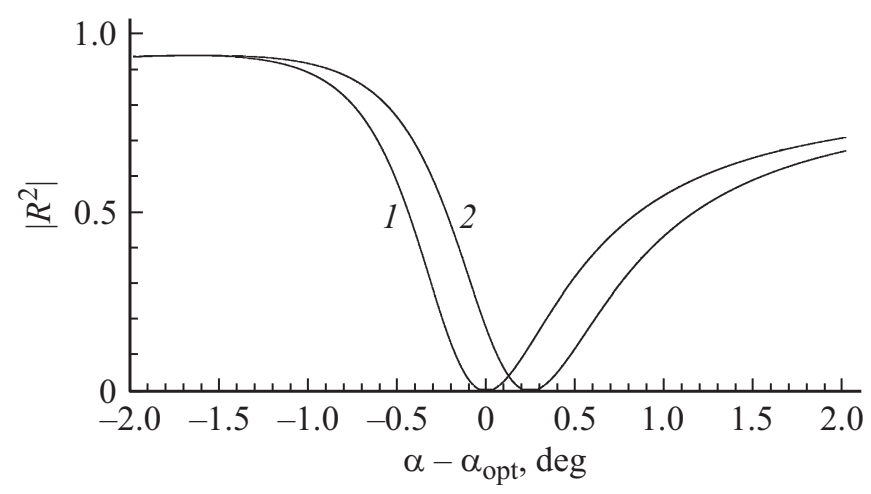

Pис. 2. Зависимости коэффициента отражения по мощности $|R|^{2}$ от разности угла падения и оптимального угла падения $\alpha_{\text {opt }}=40.98^{\circ}$. Кривая $1-$ над пленкой золота находится воздух, кривая 2 - дополнительно нанесена пленка воды толщиной $3 \mathrm{~nm}$.

свободного воздушного пространства. Параметры приведены в табл. 1 (первая строка данных). Пусть теперь на поверхность пленки нанесена тонкая пленка воды с толщиной $3 \mathrm{~nm}$ и показателем преломления $n_{w}=1.33$ на длине волны $\lambda=633 \mathrm{~nm}$. На рис. 2 показаны зависимости коэффициента отражения (по мощности) падающей со стороны призмы плоской волны от разницы угла падения $\alpha$ и оптимального угла падения $\alpha_{\text {opt }}=40.98^{\circ}$ (соответствующего минимуму отражения в отсутствие пленки воды). Показаны зависимости для свободной поверхности золота и для случая, когда на поверхности золота находится указанная пленка воды. Из результатов расчетов видно, что при появлении пленки воды минимум отражения немного, но заметно смещается на $\Delta_{s p}=0.246^{\circ}$.

Далее мы рассмотрим датчик на многослойном поверхностном волноводе и сравним смещение минимума при появлении на его свободной поверхности пленки воды с теми же параметрами. Смещение минимума даст нам представление об увеличении чувствительности многослойного датчика.

\section{Датчик на многослойном поверхностном волноводе, нахождение оптимальной конструкции}

Так же как в работе [12] мы рассмотрим в качестве основы поверхностного волновода пленку с высоким показателем преломления $n_{w g}=2.2$ (высоким по сравнению с показателем преломления призмы $n_{p}=1.6$ ). Толщину этой пленки $d_{w g}$ (рис. 3) мы будем оптимизировать ниже по специальной численной процедуре. Параллельно с пленкой с $n_{w g}=2.2$ (рис. 3) расположим пленку слабопроводящего материала (типа ITO) с диэлектрической проницаемостью $\varepsilon_{a}=3.24+i 0.002$. Мнимая часть проницаемости этой

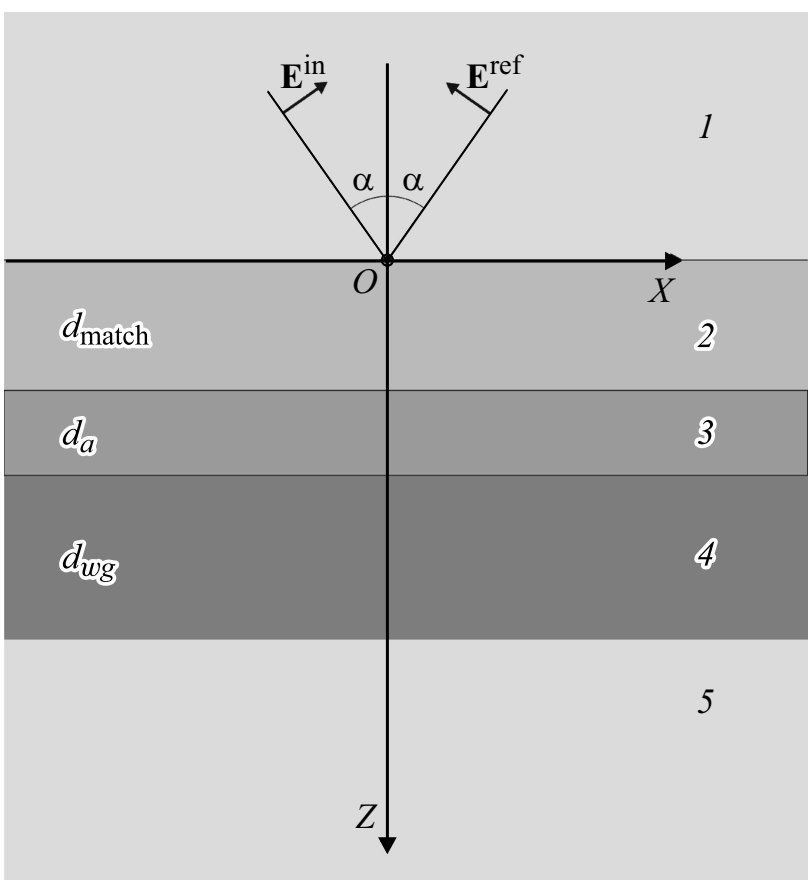

Рис. 3. Структура пленок поверхностного многослойного волновода: 1 - призма; 2 - согласующий слой с малым показателем преломления и толщиной $d_{\text {match }} ; 3-$ слабо поглощающий слой толщиной $d_{a} ; 4-$ слой с высоким показателем преломления (основной волноводный слой) толщиной $d_{w g}$; 5 - свободное пространство.

пленки и ее толщина определяют потери составного многослойного поверхностного волновода на рабочей длине волны $\lambda=633 \mathrm{~nm}$. Толщину проводящей пленки зафиксируем и примем равной $d_{a}=80 \mathrm{~nm}$. Она будет определять поглощение поверхностной волны. Наконец, между поглощающей пленкой и призмой расположим согласующую пленку с малым показателем преломления $n_{\text {match }}=1.2$, а толщину этой согласующей пленки $d_{\text {match }}$ будем подбирать специальным образом.

Оптимизация конструкции производилась по методике работы [12] или [19]. Суть метода состоит в переборе различных значений $d_{\text {match }}$ и $d_{w g}$ с малым шагом и в широком диапазоне изменений. Все остальные параметры задачи фиксируются (двухпараметрическая оптимизация). В качестве результата оптимизации находится такое сочетание размеров указанных толщин, при котором в угловом спектре отражения наблюдается наиболее острый минимум (минимум миниморум от всех угловых распределений). Опыт численной оптимизации показал, что всегда, действуя таким методом, можно найти острый минимум углового распределения. В табл. 2 приведены значения оптимальных толщин для случая свободного пространства с показателем преломления $n_{s}=1$ (воздух). 
Таблица 2

\begin{tabular}{c|c|c|c|c}
\hline № слоя & Название & Показатель преломления & Диэлектрическая проницаемость & Оптимальная толщина, пт \\
\hline 1 & Призма & $n_{p}=1.6$ & $\varepsilon_{p}=2.56$ & - \\
\hline 2 & $\begin{array}{c}\text { Согласующая } \\
\text { пленка }\end{array}$ & $n_{\text {match }}=1.2$ & $\varepsilon_{\text {match }}=1.44$ & $d_{\text {match }}=228.8$ \\
\hline 3 & $\begin{array}{c}\text { Поглощающая } \\
\text { пленка }\end{array}$ & $n_{a}=1.8+i 5.556 \cdot 10^{-4}$ & $\varepsilon_{w g}=4.84$ & $d_{a}=80$ \\
\hline 4 & $\begin{array}{c}\text { Оптически } \\
\text { плотная пленка }\end{array}$ & $n_{w g}=2.2$ & $\varepsilon_{w g}=328.2$ \\
\hline 5 & $\begin{array}{c}\text { Свободное } \\
\text { пространство }\end{array}$ & $n_{s}=1$ & -
\end{tabular}

\section{Влияние отклонения толщин пленок от оптимальных значений в датчике на многослойном поверхностном волноводе и в датчике на поверхностных плазмонах}

При конкретной реализации датчиков возникают следующие вопросы:

- Что будет с характеристиками датчиков, если толщины пленок будут изготавливаться с конечной точностью, например с точностью $\pm 1 \mathrm{~nm}$ ?

- Насколько изменится при этом глубина минимума отражения и его угловое положение?

Чтобы ответить на эти вопросы, были проведены расчеты зависимости коэффициента отражения по мощности (интенсивности) от углового отклонения от оптимального угла $\alpha_{\text {opt }}=84.8094^{\circ}$ для полученных выше оптимальных конструкций (табл. 2) с отклонениями толщин пленок на $\pm 1 \mathrm{~nm}$ (рис. $4, a$ ). Центральная кривая 1 соответствует оптимальным толщинам пленок, приведенным в табл. 2. Кривые 2 и 3 соответствуют $d_{a}-1 \mathrm{~nm}$ и $d_{a}+1 \mathrm{~nm}$, кривые 4 и 5 соответствуют $d_{w g}-1 \mathrm{~nm}$ и $d_{w g}+1 \mathrm{~nm}$ (при неизменных остальных толщинах). То есть варьировалась толщина каждой пленки в отдельности. Кривые для $d_{\text {match }} \pm 1 \mathrm{~nm}$ на рис. $4, a$ не показаны, так как смещение оказалось очень малым по сравнению с кривыми 2-4.

Из проведенных расчетов следует важный вывод: неточность порядка $1 \mathrm{~nm}$ в изготовлении пленок не скажется на работоспособности датчика. Указанная неточность приводит лишь к смещению в реальной конструкции угла, соответствующего минимуму коэффициента отражения, и незначительному уменьшению глубины минимума.

Аналогичные расчеты, проведенные для датчика на поверхностных плазмонах (табл. 1, первая строка), показали значительно меньшее влияние точности напыления пленки золота. На рис. 4, $b$ показаны зависимости коэффициента отражения по мощности от разницы угла падения $\alpha$ и угла падения $\alpha_{\text {opt }}=40.98^{\circ}$. Центральная
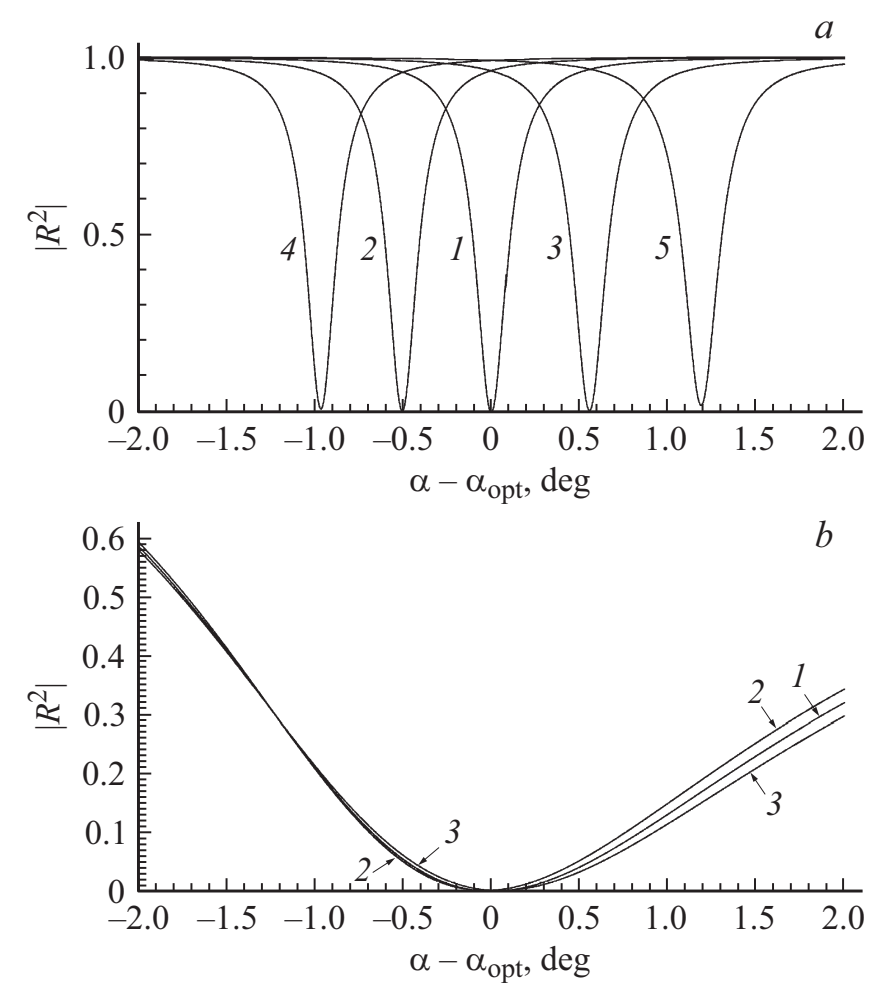

Рис. 4. (a) Коэффициент отражения по мощности $|R|^{2}$ как функция от углового отклонения от оптимального угла $\alpha_{\text {opt }}=84.8094^{\circ}$. Кривая 1 соответствует оптимальным толщинам пленок (табл. 2). Кривые 2 и 3 соответствуют $d_{a}-1 \mathrm{~nm}$ и $d_{a}+1 \mathrm{~nm}$, кривые 4 и 5 соответствуют $d_{w g}-1 \mathrm{~nm}$ и $d_{w g}+1 \mathrm{~nm}$ (при неизменных остальных толщинах). (b) Коэффициент отражения по мощности $|R|^{2}$ как функция от углового отклонения от оптимального угла $\alpha_{\text {opt }}=40.98^{\circ}$ для золотой пленки оптимальной толщины (табл. 1). Кривая 1 соответствует оптимальной толщине пленки. Кривые 2 и 3 - для толщины пленки, увеличенной и уменьшенной на $1 \mathrm{~nm}$.

кривая 1 соответствует оптимальной толщине пленки золота без отклонения. Кривые 2 и 3 - для толщины пленки, увеличенной и уменьшенной на $1 \mathrm{~nm}$ от оптимальной. Из рис. $4, b$ видно, что и для этого случая 


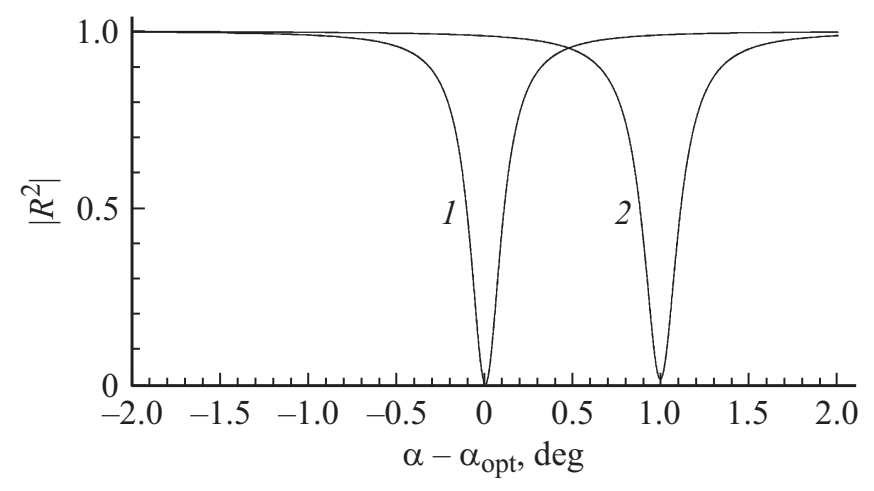

Рис. 5. Коэффициент отражения $|R|^{2}$ как функция разницы угла падения $\alpha$ и оптимального угла падения $\alpha_{\mathrm{opt}}=84.8094^{\circ}$. Кривая 1 соответствует оптимальной конструкции поверхностного волновода без пленки воды, а кривая 2 с указанной пленкой толщиной $3 \mathrm{~nm}$.

небольшие отклонения толщины пленки от оптимальной не влияют на работоспособность датчика, приводят только к небольшому угловому смещению минимума отражения и уменьшению глубины минимума.

\section{Оценка чувствительности датчика на многослойном поверхностном волноводе}

Наконец, рассмотрим, как сместится угловой минимум отражения при возникновении на свободной поверхности датчика на многослойном поверхностном волноводе пленки воды толщиной $3 \mathrm{~nm}$. Результаты расчетов показаны на рис. 5. Приведены зависимости коэффициента отражения (по мощности) падающей со стороны призмы плоской волны от разницы угла падения $\alpha$ и оптимального угла падения $\alpha_{\text {орt }}=84.8094^{\circ}$. Кривая 1 соответствует оптимальной конструкции без пленки воды, а кривая 2 - с указанной пленкой. Мы видим значительное угловое смещение минимума отражения.

Угловое смещение минимума отражения (рис. 5), возникающее при возникновении на свободной поверхности датчика пленки воды толщиной $3 \mathrm{~nm}$, равно $\Delta_{s w g}=0.991^{\circ}$. В то время как угловое смещение минимума отражения для датчика на поверхностных плазмонах (рис. 2) равно $\Delta_{s p}=0.246^{\circ}$. Следовательно, чувствительность рассматриваемого датчика на поверхностной волне в многослойной структуре равна $0.33 \mathrm{deg} / \mathrm{nm}$, что в 4 раза выше чувствительности датчика на поверхностных плазмонах $0.082 \mathrm{deg} / \mathrm{nm}$. Отметим, что такая чувствительность датчика на поверхностной волне в многослойной структуре выше, чем у любого датчика на поверхностных плазмонных волнах, чувствительность которых ограничена проводимостью металлов.

\section{Оценка минимального размера датчика вдоль направления распространения поверхностной волны}

При практической реализации каждый датчик имеет ограниченные размеры, поэтому надо учесть то обстоятельство, что поверхностная волна, возбуждаемая при резонансном отражении, распространяется на некоторое расстояние вдоль поверхности за границу падающего светового пучка (вычисления данного раздела были проделаны методами, подробно изложенными в $[11,15,16])$. При этом эта поверхностная волна излучает энергию в призму и дает вклад в угловое распределение отраженных волн. Очевидно, что размер датчика должен быть достаточно большим, чтобы вмещать большую часть поверхностной волны, включая область ее затухания.

На рис. 6, $а$ показаны распределения амплитуд составляющих электрического поля на внешней границе рассмотренной выше многослойной структуры (табл. 2) при падении со стороны призмы ограниченного светового пучка под оптимальным углом. По сути, это распределение полей поверхностной волны. Поля нормированы на амплитуду падающего из призмы светового
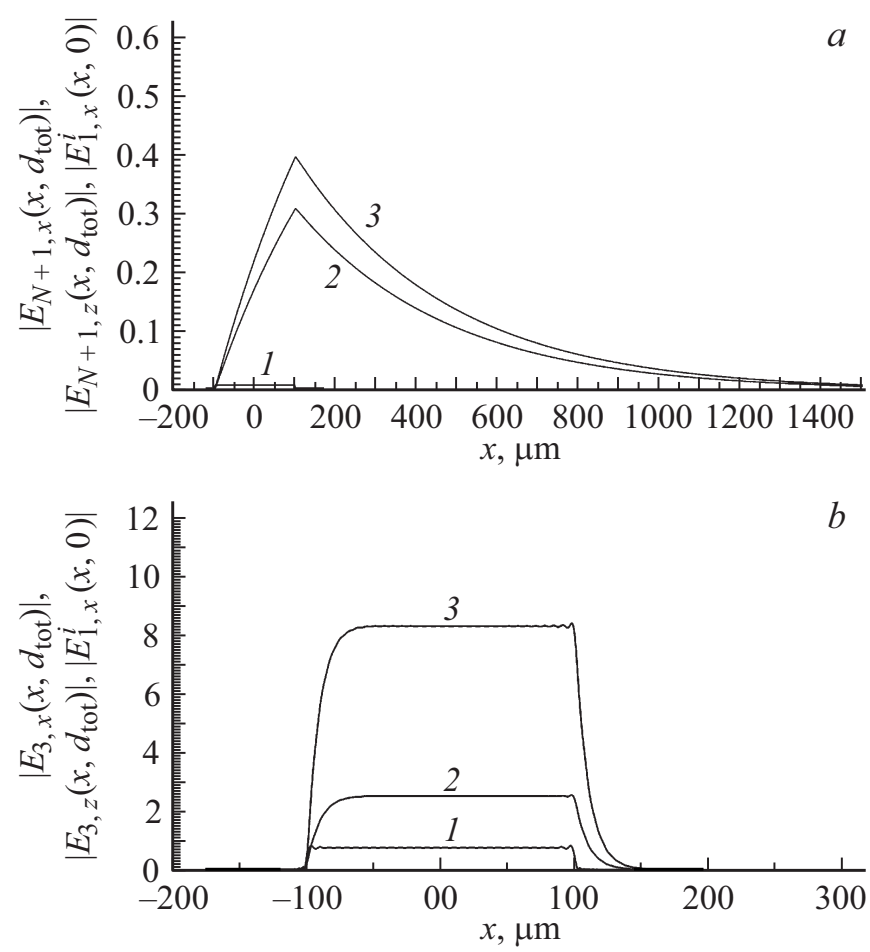

Рис. 6. (a) Распределение модуля $\left|E_{x}^{i}\right| x$-составляющей электрического поля на внутренней границе многослойного поверхностного волновода (кривая 1) и распределений модулей $\left|E_{N+1, x}\right| x$ - и $\left|E_{N+1, z}\right| z$-составляющих (кривые 2 и 3 соответственно) на внешней границе многослойного поверхностного волновода (табл. 2) при падении из призмы ограниченного светового пучка под оптимальным углом. (b) Те же распределения, но для золотой пленки (табл. 1, первая строка) при падении из призмы ограниченного светового пучка под оптимальным углом. 
пучка. Для сравнения показано также распределение $x$ составляющей амплитуды электрического поля, падающего на многослойную структуру со стороны призмы светового пучка. Из рисунка видно, что поверхностная волна довольно медленно затухает, и можно оценить минимально необходимый размер активной части поверхностного волновода в $1.5-2 \mathrm{~mm}$.

Для сравнения на рис. $6, b$ показаны те же распределения, что и на рис. 6, $a$, но проделанные для золотой пленки датчика на поверхностной плазмонной волне (табл. 1, первая строка). Из анализа рис. $6, b$ можно сделать вывод, что область засветки пучка можно сделать примерно равной $25 \mu \mathrm{m}$, при этом минимально необходимый размер активной части золотой пленки одного датчика можно сделать равной примерно $50 \mu \mathrm{m}$.

\section{Обсуждение результатов}

В результате проделанных выше исследований можно ответить на вопрос о том, как получить еще бо́льшую чувствительность датчика на многослойном поверхностном волноводе при заданных технологических возможностях тонкопленочных технологий? Ответ следующий:

1. Для увеличения чувствительности надо уменьшать поглощение в многослойном волноводе, а это достигается уменьшением толщины $d_{a}$ поглощающей пленки. Поэтому сначала надо выбрать толщину этой пленки исходя из планируемого значения чувствительности.

2. После этого надо выбрать оптимальные толщины пленок основного волновода $d_{w g}$ и согласующего слоя $d_{\text {match }}$ по методу оптимизации, описанному выше.

3. Варьированием толщин пленок в пределах точности их изготовления убеждаемся, что заметный минимум в угловом распределении отражения отчетливо наблюдается при самых неудачных технологических вариациях.

4. Далее убеждаемся, что полученная чувствительность такая, как планировалась, например, путем проведения вышеописанного теста на дополнительную пленку воды в $3 \mathrm{~nm}$.

5. Наконец, находим минимальный размер датчика в плоскости пленки. Убеждаемся, что на всем этом размере наша технология действительно способна обеспечить необходимые толщины пленок с заданной точностью. Если все эти условия выполнены, то необходимая чувствительность может быть реально достигнута при данной технологии изготовления пленок.

\section{Заключение}

Итак, на основе теории отражения светового пучка от плоскослоистой структуры с резонансным отражением и возбуждением поверхностной волны по схеме Кречмана предложен метод нахождения многослойных структур, дающих повышенную чувствительность соответствующих датчиков. В частности, предложена конкретная конструкция, чувствительность которой в 4 раза больше со- ответствующего датчика на поверхностной плазмонной волне на свободной поверхности пленки золота. Произведены оценки необходимой точности изготовления толщин пленок для получения работоспособных датчиков. Обсуждены способы увеличения чувствительности датчиков на многослойном поверхностном волноводе. Особо отметим, что по сравнению с датчиками на поверхностных плазмонных волнах использование многослойных диэлектрических структур позволяет расширить диапазон рабочих длин волн датчиков, в частности построить датчики на поверхностных волнах в ИК и УФ диапазонах.

\section{Список литературы}

[1] Homola J., Yee S.S., Gauglitz G. // Sensors and Actuators B. 1999. V. 54. P. 3.

[2] Barnes W.L., Dereux A., Ebbesen T.W. // Nature. 2003. V. 424. P. 824.

[3] Homola J. // Chem. Rev. 2008. V. 108. P. 462.

[4] Spoto G., Minunni M. // J. Phys. Chem. Lett. 2012. V. 3. P. 2682.

[5] Raether H. Surface Plasmons. Berlin: Springer-Verlag, 1988. $353 \mathrm{p}$.

[6] Barnes W.L. // J. Opt. A: Pure Appl. Opt. 2006. V. 8. P. S87.

[7] Kretschmann E., Raether H.Z. // Naturforsch A. 1968. V. 23. P. 2135.

[8] Piliarik M., Homola J. // Opt. Express. 2009. V. 17. N 19. P. 16505.

[9] Liedberg B., Nylander C., Lundstrom I. // Sensors and Actuators. 1983. V. 4. P. 299.

[10] Liedberg B., Nylander C., Lundstrom I. // Biosensors \& Bioelectronics. 1995. V. 10. P. i-ix.

[11] Петрин А.Б. // Опт. и спектр. 2018. Т. 125. № 3. С. 375.

[12] Петрин А.Б., Вольпян О.Д., Сигов А.С. // Опт. и спектр. 2017. T. 123. № 5. C. 786.

[13] Garabedian R., Gonzalez C., Richards J. et al. // Sensors and Achrators A. 1994. V. 43. P. 202.

[14] Yeatman E.M. // Biosensors \& Bioelectronics. 1996. V. 11. P. 635.

[15] Петрин А.Б. // Опт. и спектр. 2018. Т. 125. № 6. С. 830.

[16] Петрин А.Б. // Квант. электрон. 2019. Т. 49. Т. 3. С. 258.

[17] Airoudj A., Debarnot D., Beche B., Poncin-Epaillard F. // Anal. Chem. 2008. V. 80. P. 9188.

[18] Nesterenko D.V., Hayashi S., Sekkat Z. // J. Opt. 2016. V. 18. P. 065004.

[19] Петрин А.Б., Вольпян О.Д., Сигов А.С. // ЖТФ. 2018. Т. 88. 3. C. 433. 